\title{
AVALIAÇÃO DA QUALIDADE DO PLANEAMENTO DO 'MODELO DE AVALIAÇÃO E INTERVENÇÃO FAMILIAR INTEGRADA' PARA OS CENTROS DE APOIO FAMILIAR E DE ACONSELHAMENTO PARENTAL
}

\author{
Ana Melo ${ }^{1}$ \\ Madalena Alarcão ${ }^{2}$
}

Resumo: Este artigo apresenta os resultados da avaliação da qualidade do planeamento de um Modelo de Avaliação e Intervenção Familiar Integrada (MAIFI) tendo em vista a sua implementação em Centros de Apoio Familiar e Aconselhamento Parental. Utilizando-se uma adaptação da Técnica de Grupo Nominal, um painel de especialistas avaliou a proposta apresentada, quer no que diz respeito ao MAIFI, quer no que toca ao perfil de competências dos profissionais que o venham a implementar e ao plano de formação dos mesmos. As médias das avaliações quantitativas indicam resultados bastante satisfatórios considerando a relevância, a qualidade do enquadramento conceptual e da definição de objectivos, a adequação às características da população-alvo, a qualidade dos métodos propostos, dos recursos necessários e dos procedimentos de avaliação das propostas. Foi realizada uma análise do conteúdo da reunião de avaliação, sendo apresentadas e

\footnotetext{
${ }^{1}$ Bolseira da Fundação para a Ciência e Tecnologia, Faculdade de Psicologia e de Ciências da Educação da Universidade de Coimbra

${ }^{2}$ Faculdade de Psicologia e de Ciências da Educação da Universidade de Coimbra Contactos para correspondência: Ana Melo, Rua Camilo Vaz, n. ${ }^{\circ} 45.3^{\circ}$ Dto. Centro, 4430-686 Vila Nova de Gaia, Portugal. Email: anamelopsi@gmail.com Agradece-se, à Dra. Dora Pereira, em representação da Associação "Chão dos Meninos", à Dra. Laura Teles, em representação do Instituto da Solidariedade Social, ao Dr. António Pinto de Matos, em representação da Confederação Nacional das Instituições de Solidariedade, à Professora Doutora Teresa Ribeiro, da Faculdade de Psicologia e Ciências da Educação da Universidade de Lisboa, à Professora Doutora Liliana Sousa, da Universidade de Aveiro, ao Doutor William Madsen, Director do Programa de Terapia Narrativa do Family Institute of Cambridge nos Estados Unidos da América, e ao Dr. Ramon Rojano, Director de Serviços Humanos do Wake County, North Carolina, nos Estados Unidos da América, por aceitarem o convite para integrar o painel de especialistas.

O presente trabalho foi desenvolvido no âmbito de uma bolsa de doutoramento financiada pela Fundação para a Ciência e Tecnologia (SFRH/BD/39912/2007), atribuída à primeira autora.
} 
discutidas as dúvidas colocadas pelos especialistas, as avaliações positivas e negativas e as sugestões de melhoramento.

Palavras-chave: avaliação de planeamento de programa, crianças e jovens em risco ou perigo, famílias multi-desafiadas, intervenção familiar, perfil de competências profissionais, formação

The Integrated Family Assessment and Intervention Model (IFAIM): Evaluation of a proposal to be implemented in Centers for Family Support and Parental Counseling (Abstract): This article reports the results of a quality planning evaluation of a proposal of the Integrated Family Assessment and Intervention Model (IFAIM) to be implemented in Centers for Family Support and Parental Counseling.

Through an adaptation of the Nominal Group Technique, an expert panel performed quantitative and qualitative evaluations of a proposal describring IFAIM, a profile of competencies for its professionals and the respective training programme. The calculation of the mean score for each dimension yielded quite satisfactory results considering the relevance, the conceptual background and the definition of objectives, necessary resources and evaluation procedures of the model. A content analysis of the meeting was conducted. The results regarding the experts' questions, their positive and negative evaluations as well as suggestions for improvement are reported and discussed.

Keywords: programme planning evaluation, at-risk children and youth, multi-challenged families, family intervention, professional skills' profile, training

\section{Introdução}

Os serviços de apoio familiar, destinados a famílias com crianças e jovens em risco ou em perigo, têm uma história recente em Portugal. Os Centros de Apoio Familiar e Aconselhamento Parental (CAFAP) são definidos como uma "resposta social, desenvolvida através de um serviço, vocacionada para o estudo e prevenção de situações de risco social e para o apoio a crianças e jovens em situação de perigo e suas famílias, concretizado na sua comunidade através de equipas multidisciplinares" (DGSSFC, 2006, p. A 3.1). Tradicionalmente, os serviços de apoio familiar baseiam-se na ideia de que a família deve ser apoiada para cumprir eficazmente as suas funções. Perante condições de perigo, nomeadamente de mautrato, devem ser desenvolvidas intervenções que permitam manter a criança no seio familiar, desde que se reduzam ou eliminem as circunstâncias de risco de mautrato (Armstrong \& Hill, 2001; Hoagwood, 2005; Manalo \& Meezan, 2000). Estes pro- 
cessos decorrem, frequentemente, em contextos de coacção e conduzem à emissão de pareceres que podem influenciar, muitas vezes de forma decisiva, a vida das famílias (Cirillo \& Di Blasio, 1997; Walsh, 2006). As famílias acompanhadas pelos serviços sociais são, frequentemente, 'multi-desafiadas', colocando uma série de dificuldades aos profissionais. Os múltiplos desafios dificultam os esforços de preservação ou reunificação familiar.

O Modelo de Avaliação e Intervenção Familiar Integrada (MAIFI, Melo, 2008) foi desenhado para dar resposta às necessidades de avaliação e intervenção junto destas famílias, multi-desafiadas e com crianças e jovens em situação de risco psicossocial ou perigo (particularmente de mau trato físico, psicológico ou negligência), acompanhadas pelos Centros de Apoio Familiar e Aconselhamento Parental (Melo \& Alarcão, 2009a). Um dos aspectos mais distintivos do MAIFI, tal como detalhado noutro lugar (Melo \& Alarcão, 2010b), é o seu carácter integrador. O MAIFI caracteriza-se, não só pela combinação de abordagens tradicionalmente separadas (e.g., trabalho clínico, social, educativo, comunitário e forense), mas também por integrar contributos de diferentes modelos e abordagens de intervenção familiar e parental. Esta integração decorre sob a alçada de um pensamento sistémico (Carr, 2006; Dallos \& Urry, 1999; Vetere \& Dallos, 2003) e de uma postura de base colaborativa (Hoffman, 1998; Madsen, 2007). O MAIFI reflecte uma forte influência de abordagens colaborativas, narrativas e centradas nas forças e soluções (Andersen, 1995; Anderson \& Goolishian, 1992; DeShazer, 1991; Friedman, 1993; Saleebey, 2002; Sluzki, 1992; White, 2007) e da sua aplicação ao trabalho clínico e social em situações de risco e perigo, particularmente de mau trato (Berg \& Kelly, 2000; De Jong \& Miller, 1995; Madsen, 2007; Turnell \& Edwards, 1999). Trata-se, ainda, de um modelo multisistémico (Boyd-Franklin \& Bry, 2000), focado na família e orientado por modelos de resiliência familiar (Walsh, 2006), que valoriza o trabalho no domicílio (Boyd-Franklin \& Bry, 2000; Woodford, Bordeau, \& Alderfer, 2006). Desta forma, o MAIFI procura colmatar uma lacuna no panorama português no que diz respeito à existência de intervenções familiares especializadas, adequadas às múltiplas necessidades de famílias multi-desafiadas (Melo \& Alarcão, 2010a; Sousa, 2005) e especialmente adaptadas a situações de pobreza (Frankel \& Frankel, 2006; Rojano, 2004).

Organiza-se em cinco fases, com objectivos, orientações, procedimentos e instrumentos de trabalho próprios. O quadro 1 apresenta um resumo dos objectivos específicos associados a cada etapa de implementação do modelo. 
Quadro 1. Objectivos específicos de cada etapa de implementação do MAIFI

\begin{tabular}{|c|c|}
\hline Etapa do MAIFI & Objectivos \\
\hline Encaminhamento & $\begin{array}{l}\text { - Recolher informação sobre a situação da família } \\
\text { - Definir os contornos da fase de avaliação, clarificar os papéis, res- } \\
\text { ponsabilidades e modos de articulação entre o CAFAP e outros pro- } \\
\text { fissionais } \\
\text { - Elaborar hipóteses de trabalho compreensivas } \\
\text { - Definir o contexto inicial e os elementos da equipa que vão estar } \\
\text { envolvidos }\end{array}$ \\
\hline Acolhimento & $\begin{array}{l}\text { - Criar uma relação de colaboração com a família } \\
\text { - Negociar e definir os contornos do pedido e da relação entre a famí- } \\
\text { lia, o CAFAP e outros profissionais/organizações } \\
\text { - Definir estratégias de trabalho e negociar objectivos e procedimen- } \\
\text { tos para o período de avaliação }\end{array}$ \\
\hline Avaliação & $\begin{array}{l}\text { - Avaliar as principais forças e vulnerabilidades da família relativa- } \\
\text { mente ao risco de maus tratos futuros e às possibilidades de ajusta- } \\
\text { mento e desenvolvimento positivo da criança e da família } \\
\text { - Construir hipóteses sobre os factores que contribuíram para a emer- } \\
\text { gência e manutenção dos problemas e sobre os factores constrangedo- } \\
\text { res ou facilitadores da construção de condições de segurança e do } \\
\text { desenvolvimento das visões, direcções e intenções preferidas de vida } \\
\text { da família } \\
\text { - Aumentar a disponibilidade da família para a mudança } \\
\text { - Avaliar o potencial de mudança da família, avançando com prog- } \\
\text { nósticos } \\
\text { - Definir possibilidades e estratégias de suporte para a mudança e } \\
\text { elaborar um relatório de avaliação }\end{array}$ \\
\hline $\begin{array}{l}\text { Suporte para a } \\
\text { Mudança }\end{array}$ & $\begin{array}{l}\text { - Aumentar a segurança e bem-estar dos elementos da família, em } \\
\text { particular da criança } \\
\text { - Aumentar a percepção da família de viver de acordo com as visões } \\
\text { preferidas de si e as suas direcções preferidas de vida } \\
\text { - Aumentar a capacidade da família de exercer o seu direito à liberdade } \\
\text { - Aumentar processos de resiliência familiar (a nível da dimensão das } \\
\text { crenças familiares, processos organizacionais e capacidade de comu- } \\
\text { nicação e resolução de problemas) e individual e promover a capaci- } \\
\text { dade da família de lidar com os múltiplos desafios com que se con- } \\
\text { fronta } \\
\text { - Aumentar a qualidade das condições sociais de vida das famílias } \\
\text { e/ou a sua capacidade de as melhorarem } \\
\text { - Aumentar competências parentais (ao nível dos cuidados básicos, } \\
\text { segurança afectiva, orientação e estabelecimento de limites, seguran- } \\
\text { ça e estimulação) facilitadoras de um desenvolvimento positivo das } \\
\text { crianças e jovens }\end{array}$ \\
\hline
\end{tabular}


- Diminuir os problemas e mau estar percebidos pelas famílias - Aumentar o bem-estar integrado (físico, psicológico, social) dos elementos das famílias

Follow-up e Fina- - Promover a manutenção das mudanças

lização - Avaliar a pertinência e eficácia das mudanças verificadas face aos objectivos definidos

O carácter colaborativo do MAIFI e o cuidado na organização dos processos de avaliação permitem que o trabalho seja orientado para a produção e organização de informação rigorosa e útil, que facilite o processo de tomada de decisão por parte do sistema de promoção e protecção (ex: CPCJ, Tribunais). O modelo focaliza-se na facilitação da mudança, apoiando a família, desde os primeiros contactos, para que: a) construa condições de segurança e bem-estar para a criança; b) persiga as suas visões e direcções preferidas de vida; e c) exerça o seu direito à liberdade (entendido como a capacidade de obter e produzir informação sobre si própria, o mundo e a sua relação com ele, assim como a real capacidade de fazer escolhas e de assumir a responsabilidade pelas mesmas).

A importância dada ao trabalho no espaço domiciliário e comunitário associa-se a um conjunto de factores tais como: a) o respeito e a compreensão pelo enquadramento ecossistémico da vida familiar (Boyd-Franklin \& Bry, 2000; Minuchin, Colapinto, \& Minuchin, 2007); b) a combinação de um olhar clínico com um trabalho de cariz social (Rojano, 2004); c) uma atenção especial a barreiras e obstáculos que possam dificultar o envolvimento das famílias (Cunningham \& Hengeller, 1999); e d) a valorização das forças, competências, recursos e redes naturais da família na facilitação do processo de mudança (Saleebey, 2002).

O MAIFI foi desenvolvido de modo a poder ser manualizado, disseminado e avaliado em termos de processo e de resultados, tendo sido definidos um perfil de competências e um plano de formação e acompanhamento dos profissionais que venham a implementá-lo. As competências dos profissionais dividem-se em competências teóricas, de conceptualização de caso e de relação profissional-família, e competências práticas. As primeiras estão estritamente relacionadas com o carácter sistémico e ecológico, colaborativo, narrativo e centrado nas forças e competências, subjacente ao MAIFI, bem como com as exigências de avaliação e intervenção em contextos de coacção e situações de risco e perigo, particularmente de maus tratos físicos, psicológicos e negligência. As segundas, subdividem-se em diferentes categorias, conforme se referem a: a) competências básicas de gestão das sessões, das quais depende, em larga medida, o carácter colaborativo, e centrado nas for- 
ças, do MAIFI; b) competências relacionadas com a fase de acolhimento e avaliação; c) competências relacionadas com a fase de suporte para a mudança, fortemente associadas a práticas narrativas, modelos de competência e centradas nas soluções; d) competências associadas à gestão das sessões em contexto domiciliário e comunitário; e) competências associadas à gestão colaborativa das sessões com outros profissionais e/ou outros elementos significativos para a família.

Nas últimas décadas, a avaliação de programas tem ganho uma maior notoriedade. Trata-se de uma categoria específica de investigação, dirigida para um propósito particular, geralmente focalizado no desempenho de um serviço ou de uma modalidade de intervenção (Robson, 1993). A sua relevância prende-se, em larga medida, com o imperativo de se apresentarem resultados da eficácia das intervenções e dos programas psicossociais, de modo a legitimar-se a sua adopção e financiamento (Pecora, Seelig, Zirps, \& Davis, 1996; Posavac \& Carey, 2003; Rossi, Lipsey, \& Freeman, 2004).

Os objectivos das avaliações de programas podem ser diversos e, como propõe Patton (1997), podem implicar não apenas um julgamento sobre o seu valor, mas visar também o seu melhoramento ou, ainda, a produção de conhecimento científico.

Muito embora as técnicas de investigação e métodos utilizados tendam a não ser substancialmente diferentes de outras formas de investigação (Robson, 1993), a avaliação de programas diferencia-se pela natureza das questões colocadas, pelo seu propósito e pela utilização, normalmente mais imediata e focalizada, do resultado da investigação (Rossi et al., 2004). Desde o desenvolvimento à disseminação de um programa, podem ter lugar diferentes tipos de avaliação, orientados para responder a diferentes categorias de questões, cujas respostas podem informar acções particulares por quem desenvolve, adopta, implementa e financia um programa, ou mesmo por quem dele recebe serviços (Herman, Morris, \& Fitz-Gibbon, 1987; Rossi et al., 2004).

A avaliação de um programa deve iniciar-se com a avaliação do seu planeamento (Kröger, Winter \& Shaw, 1998), podendo incluir a avaliação de necessidades e a avaliação do desenho e teoria do programa (Rossi et al., 2004; Pecora et al., 1996; Posavac \& Carey, 2003).

O recurso a métodos de avaliação por painéis de especialistas, embora pouco estudado, parece apresentar vantagens e limitações (Averch, 2004; Patton, 2002; Posavac \& Carey, 2003). Por um lado, trata-se de uma metodologia de baixo custo que pode, na realidade, ser a única disponível para a avaliação de aspectos particulares, como o enquadramento teórico do programa (Averch, 2004; Posavac \& Carey, 2003; Rossi et al., 2004). Por outro lado, esta avaliação está fortemente dependente das qualificações dos especialistas envolvidos, das suas características e preferências pessoais, levan- 
tando-se, assim, algumas ameaças à sua validade e fidelidade (Averch, 2004; Patton, 2002). Algumas técnicas de negociação de consenso têm vindo a ser aplicadas aos métodos de avaliação por painéis de especialistas, não apenas para aumentar a fidelidade dos resultados, quando o consenso é uma dimensão valorizada, mas, também, para estimular a diversidade de pontos de vista e de soluções, quando a avaliação visa o melhoramento de um programa (Jones, 1995; McDonald, Bammer \& Deane, 2009).

Considerando que o Modelo de Avaliação e de Intervenção Familiar Integrada se encontra numa fase inicial de desenvolvimento, importa avaliar a qualidade do seu planeamento, tendo em vista o seu melhoramento e um melhor aproveitamento dos recursos associados à sua operacionalização.

\section{Objectivos}

O objectivo geral do presente estudo é avaliar a qualidade do planeamento do MAIFI no que diz respeito à sua adequação relativamente: ao fenómeno-alvo e necessidade de intervenção, ao quadro conceptual e aos objectivos definidos, à população-alvo, aos métodos, aos recursos e aos procedimentos de avaliação (Hoffman, 2005; Kröger, Winter, \& Shaw, 1998; Posavac \& Carey, 2003).

Este estudo pretende, ainda, avaliar a qualidade da proposta do perfil de competências dos profissionais do MAIFI e do plano de formação e acompanhamento dos mesmos.

\section{Metodologia}

\section{Participantes}

Participaram neste estudo sete especialistas (E1 a E7), das áreas do apoio, avaliação e intervenção junto de famílias com crianças e jovens em situação de risco. Pretendia-se que neste painel fossem representados os seguintes pontos de vista: do profissional de terreno (E1); do académico/investigador nacional (E2 e E3); da gestão das organizações nacionais (E4); do académico/investigador internacional (E5 e E6) e do político/financiador (E7). E1 foi seleccionado pela sua experiência prática de trabalho no primeiro CAFAP do país, reconhecido pela qualidade da sua preparação técnica e científica. E2 e E3 foram convidados pela sua produção académica e científica, pela experiência de ensino/formação no domínio da intervenção familiar e, no caso de E3, também pela experiência de investigação com famílias multi-problemáticas pobres. E4 foi seleccionado por uma confederação de instituições nacionais de solidariedade social, a quem foi dirigido o convite, uma vez que é nestas instituições que os CAFAP habitualmente se integram. E5 e E6 
foram seleccionados pela sua produção académica e científica, bem como pela experiência prática e de formação, no domínio da intervenção familiar em serviços sociais focados na família, particularmente em famílias pobres e multi-desafiadas. Ambos são proponentes de programas de intervenção focados na família (E5 e E6), de cariz comunitário (E6) e colaborativo (E5). E5 apresenta, ainda, experiência na gestão de serviços sociais e de saúde. E7 foi indicado pelo organismo público que, em Portugal, regula e financia as respostas sociais, entre as quais se encontram os CAFAP.

\section{Medidas}

Para a avaliação das propostas foram usadas duas grelhas de avaliação, construídas especificamente para o presente estudo. A grelha de avaliação da qualidade do planeamento do MAIFI contempla 5 dimensões (quadro 2): avaliação relativa ao fenómeno e necessidade (3 itens), quadro conceptual/objectivos definidos (4 itens), população-alvo (3 itens), adequação dos métodos ( 3 itens), adequação dos recursos ( 3 itens) e adequação dos procedimentos avaliativos (5 itens), num total de 21 itens (quadro 2), cotados numa escala de Likert de 5 pontos, a variar entre o nada satisfatório/adequado e o muito satisfatório/adequado. A grelha de avaliação da adequação do perfil de competências, da qualidade do plano de formação e do acompanhamento dos profissionais compreende 6 itens (quadro 4), cotados segundo a mesma escala.

\section{Procedimento}

Cada participante recebeu, para análise, um documento com a descrição detalhada da proposta do Modelo de Avaliação e Intervenção Familiar Integrada (Melo, 2008), juntamente com o seu enquadramento, fundamentação teórica e proposta de procedimentos de avaliação. Recebeu, ainda, a proposta de descrição e fundamentação do perfil de competências do profissional, do plano de formação e acompanhamento dos profissionais e respectivas grelhas de avaliação. $\mathrm{O}$ documento enviado continha uma terceira proposta relativa a um modelo global de organização para os CAFAP, e respectiva grelha de avaliação, cuja descrição e avaliação são apresentadas noutro local (Melo \& Alarcão, 2009b). Os participantes receberam instruções para analisarem o documento, registarem dúvidas ou comentários e preencherem, individualmente, as grelhas de avaliação. Foi solicitado que participassem numa reunião posterior, que decorreu num registo presencial e on-line. Os elementos E5, E6 e E7 participaram através de vídeo-conferência e os restantes encontraram-se reunidos, presencialmente, com os dois autores, durante, aproximadamente, 2 horas e 14 minutos. Foi utilizado um procedimento adaptado da Técnica de Grupo Nominal para dinamização da reunião (Jones, 1995; McDonald, Bammer, \& Deane, 2009). 
Num primeiro momento, os participantes foram convidados a colocar dúvidas e questões suscitadas pela leitura do documento, bem como reaç̧ões gerais à proposta. A primeira autora foi respondendo às questões colocadas $\mathrm{e}$ clarificando alguns aspectos. Os especialistas reviram, então, as suas pontuações, quando consideraram adequado. Num segundo momento, cada participante foi convidado a ditar, para cada questão, e sequencialmente, as suas pontuações, que foram registadas pela segunda autora. Muito embora o protocolo inicial tivesse previsto convidar os participantes a negociarem uma pontuação final por consenso, o painel considerou que tal seria excessivamente moroso e de utilidade reduzida se não se verificasse grande flutuação nas pontuações, sugerindo que fosse calculada a média das suas pontuações. Não se verificaram diferenças superiores a 2 pontos entre as pontuações. Acordou-se que cada especialista justificaria as pontuações inferiores a 4, apresentando sugestões para melhoramento, e partilharia as suas avaliações qualitativas. A reunião foi gravada em áudio para posterior análise de conteúdo (Coffrey \& Atkinson, 1996; Silverman, 2006).

A sessão foi transcrita, integral e literalmente. Num primeiro momento, o texto foi marcado com cores diferentes conforme se reportava a questões ou comentários dos especialistas, a respostas dos investigadores, a conversa social, a resolução de dificuldades técnicas na videoconferência, a conteúdos relacionados com a discussão da metodologia adoptada ou às pontuações para cada item das duas grelhas de avaliação. Uma vez que a mesma reunião incluía a discussão de uma proposta não incluída neste estudo, seleccionou-se, para esta análise, apenas o texto relativo às questões, comentários e avaliações dos especialistas para a qualidade de planeamento do MAIFI, de adequação do perfil de competências dos profissionais, da qualidade do plano de formação e do acompanhamento dos mesmos. A primeira autora realizou uma leitura do texto seleccionado tendo em vista a criação de categorias gerais para classificação dos temas das intervenções dos especialistas. Foram identificadas as seguintes categorias: a) comentários gerais positivos; b) comentários gerais negativos; c) dúvidas; d) avaliações positivas específicas; e) avaliações negativas específicas; f) sugestões de melhoramento. $\mathrm{O}$ texto foi marcado consoante se relacionava com a proposta do MAIFI ou com o perfil de competências e plano de formação dos profissionais e analisado separadamente. O texto foi recortado e colado de modo a corresponder a cada uma das categorias indicadas para cada uma das propostas. Numa fase seguinte, o primeiro autor procurou identificar, dentro de cada categoria, temas das intervenções dos especialistas, registando-se os elementos que os apresentaram e aqueles que os apoiaram, quando aplicado, como forma de avaliação da concordância entre especialistas. $\mathrm{O}$ procedimento foi repetido pelo segundo autor para cálculo do acordo entre-cotadores e os resultados foram discutidos de modo a obter-se um consenso. Foi obtido um acordo de $88,5 \%$ na identificação de conteúdos 
relativos à proposta do MAIFI e de $66 \%$ naqueles relativos à proposta de perfil de competências e plano de formação de profissionais.

\section{Resultados}

Os resultados da avaliação quantitativa da qualidade do planeamento do MAIFI indicam, conforme apresentado no quadro 2, pontuações médias das dimensões a variar entre 3.95, para a avaliação relativa à adequação dos recursos, e 4.74 para a avaliação relativa ao fenómeno.

Quadro 2. Dimensões e questões para avaliação da qualidade de planeamento do MAIFI, pontuações médias, mínimas e máximas

\begin{tabular}{lccc}
\hline Dimensões de avaliação e questões formuladas & $\begin{array}{c}\text { Pontua- } \\
\text { ção } \\
\text { média }\end{array}$ & $\begin{array}{c}\text { Pon- } \\
\text { tuação } \\
\text { mínima }\end{array}$ & $\begin{array}{c}\text { Pon- } \\
\text { tuação } \\
\text { máxi- } \\
\text { ma }\end{array}$ \\
\hline A. Fenómeno e necessidades & 4.74 & 5 & 5 \\
\hline $\begin{array}{l}\text { O modelo é relevante considerando o nível de } \\
\text { importância/gravidade do fenómeno? }\end{array}$ & 5 & 5 & 5 \\
$\begin{array}{l}\text { A avaliação da necessidade de intervenção sobre o } \\
\text { fenómeno é pertinente? }\end{array}$ & 5 & 5 & 5 \\
$\begin{array}{l}\text { O modelo relaciona-se adequadamente com outras } \\
\text { intervenções que estejam a ser desenvolvidas ou } \\
\text { planeadas para dar resposta ao fenómeno-alvo? }\end{array}$ & 4 & 3 & \\
\end{tabular}

\begin{tabular}{|c|c|c|c|}
\hline B. Quadro conceptual/objectivos definidos & 4.46 & & \\
\hline $\begin{array}{l}\text { 4. O modelo é adequado relativamente aos pressupos- } \\
\text { tos teóricos e conceptuais que o enquadram? }\end{array}$ & 5 & 5 & 5 \\
\hline $\begin{array}{l}\text { 5. O modelo tem em consideração o que se sabe que } \\
\text { funciona relativamente ao fenómeno em questão? }\end{array}$ & 4.43 & 4 & 5 \\
\hline $\begin{array}{l}\text { 6. O modelo tem em consideração os factores explica- } \\
\text { tivos ou mediadores do aparecimento ou manuten- } \\
\text { ção do fenómeno? }\end{array}$ & 4 & 4 & 4 \\
\hline $\begin{array}{l}\text { 7. Os objectivos definidos são pertinentes e congruen- } \\
\text { tes com o enquadramento conceptual/teórico do } \\
\text { modelo? }\end{array}$ & 4.43 & 4 & 5 \\
\hline
\end{tabular}




\begin{tabular}{|c|c|c|c|}
\hline C. População-alvo & 4.24 & & \\
\hline $\begin{array}{l}\text { 8. O processo de recrutamento da população-alvo é } \\
\text { adequado? }\end{array}$ & 4.28 & 4 & 5 \\
\hline $\begin{array}{l}\text { 9. As estratégias de manutenção da população-alvo na } \\
\text { intervenção são adequadas? }\end{array}$ & 4.28 & 4 & 5 \\
\hline $\begin{array}{l}\text { 10. O modelo é adequado às características da popula- } \\
\text { ção-alvo? }\end{array}$ & 4.14 & 4 & 5 \\
\hline
\end{tabular}

\begin{tabular}{|c|c|c|c|}
\hline D. Adequação dos métodos & 4.19 & & \\
\hline $\begin{array}{l}\text { 11. As estratégias e componentes do modelo são ade- } \\
\text { quadas? }\end{array}$ & 4.28 & 4 & 5 \\
\hline $\begin{array}{l}\text { 12. As estratégias e componentes do modelo têm em } \\
\text { conta a literatura sobre a eficácia das mesmas? }\end{array}$ & 4.28 & 4 & 5 \\
\hline $\begin{array}{l}\text { 13. A distribuição temporal das estratégias e componen- } \\
\text { tes é adequada? }\end{array}$ & 4 & 4 & 4 \\
\hline
\end{tabular}

\begin{tabular}{|c|c|c|c|}
\hline E. Adequação dos recursos & 3.95 & & \\
\hline $\begin{array}{l}\text { 14. Os recursos humanos propostos são adequados em } \\
\text { termos de número e qualificação? }\end{array}$ & 4.14 & 3 & 5 \\
\hline $\begin{array}{l}\text { 15. O modelo é aplicável do ponto de vista dos recursos } \\
\text { financeiros? }\end{array}$ & 3.85 & 3 & 5 \\
\hline $\begin{array}{l}\text { 16. O modelo tem em consideração possíveis barreiras e } \\
\text { as propostas para as ultrapassar são adequadas? }\end{array}$ & 3.85 & 3 & 5 \\
\hline
\end{tabular}

\begin{tabular}{|c|c|c|c|}
\hline F. Adequação dos procedimentos avaliativos & 4.31 & & \\
\hline $\begin{array}{l}\text { 17. Os procedimentos de avaliação de processo propos- } \\
\text { tos são adequados e exequíveis tendo em conta a sua } \\
\text { implementação no terreno? }\end{array}$ & 4.28 & 3 & 5 \\
\hline $\begin{array}{l}\text { 18. Os procedimentos de avaliação permitem satisfazer as } \\
\text { necessidades de avaliação dos profissionais do terreno? }\end{array}$ & 4.28 & 4 & 5 \\
\hline $\begin{array}{l}\text { 19. Os procedimentos de avaliação permitem satisfazer } \\
\text { as necessidades de avaliação dos órgãos de gestão } \\
\text { das organizações? }\end{array}$ & 4.57 & 4 & 5 \\
\hline $\begin{array}{l}\text { 20. Os procedimentos de avaliação permitem satisfazer } \\
\text { as necessidades de avaliação dos financiadores? }\end{array}$ & 4.14 & 3 & 4 \\
\hline $\begin{array}{l}\text { 21. Os procedimentos de avaliação permitem satisfazer } \\
\text { as necessidades de avaliação de eventuais parceiros } \\
\text { locais? }\end{array}$ & 4.28 & 4 & 5 \\
\hline
\end{tabular}


Só a avaliação relativa à adequação dos recursos pontua abaixo de 4 . No Quadro 3 são apresentados os temas das intervenções dos especialistas e referido se os investigadores responderam às questões dos participantes, fundamentando a sua posição. Conforme pode confirmar-se, o tema dos custos específicos do modelo e da relação custo-benefício foi o único ao qual as investigadoras não deram resposta, sendo este um tema que levantou dúvidas por parte dos dois especialistas que representam instituições que gerem ou financiam as respostas sociais em que o MAIFI poderá ser inserido.

Da análise de conteúdo foi possível constatar que a maioria dos especialistas foi elogiosa relativamente à proposta do MAIFI e expressou expectativas positivas sobre o desenvolvimento do mesmo (e.g., "a minha avaliação global é muito positiva", "acho que está aqui uma enorme quantidade de trabalho" (...) "acho que esta é uma possibilidade excitante"). A inovação e o carácter organizador do modelo, para o trabalho das instituições sociais, são referidos como pontos positivos gerais, apoiados por mais do que um especialista (e.g., "acho que é um trabalho fundamental, pelo menos é raríssimo e é algo fundamental, qualquer organização tem os seus procedimentos organizados, vamos lá, menos estas instituições mais de apoio social"; "aponta caminhos"). O carácter integrador do modelo foi também realçado ("parece que se baseia num grande número de fontes para montar um modelo"; "é baseado na contribuição de muitas pessoas e não está baseado no modelo médico [...] pode encontrar-se flexibilidade no conceito global").

O carácter colaborativo do modelo, a sua focalização na família e não apenas na criança, bem como a adopção de uma linguagem qualificadora da família, foram aspectos valorados como muito positivos (e.g., "apreciei realmente (...) o foco de trabalhar realmente em colaboração com as famílias em vez de partir da expertise profissional"). A apreciação menos positiva reporta-se, à semelhança da valoração quantitativa, aos custos financeiros associados à aplicação do MAIFI (e.g., "o modelo será aplicável do ponto de vista dos recursos financeiros?"; "o modelo está bem pensado mas (...) implica muitos recursos humanos"). O especialista E1 realçou, contudo, os ganhos que poderão ser obtidos, no futuro, se se conseguirem, em menos tempo, avaliações mais fidedignas e, no quadro do apoio às famílias que demonstram potencial de mudança, menos necessidade de acolhimento institucional das crianças (e.g., "há um gasto enorme em institucionalização. (...) está em causa a possibilidade de [diminuir] custos investindo na prevenção primária e secundária"). 
Quadro 3. Resultados da avaliação qualitativa da proposta relativa ao MAIFI

\begin{tabular}{|c|c|c|c|c|c|}
\hline $\begin{array}{l}\text { Dimensões de } \\
\text { avaliação }\end{array}$ & $\begin{array}{l}\text { Conteúdos das dúvidas } \\
\text { e comentários }\end{array}$ & $\begin{array}{l}\text { Resposta às } \\
\text { dúvidas } \\
\text { Sim/Não }\end{array}$ & $\begin{array}{l}\text { Conteúdos de } \\
\text { avaliações nega- } \\
\text { tivas }{ }^{1}\end{array}$ & $\begin{array}{c}\text { Conteúdos de } \\
\text { avaliações positi- } \\
\text { vas }^{1}\end{array}$ & $\begin{array}{c}\text { Conteúdos das sugestões } \\
\text { de melhoramento }\end{array}$ \\
\hline $\begin{array}{l}\text { Generalidade da } \\
\text { proposta }\end{array}$ & - & - & $\begin{array}{l}\text {-Extensão do } \\
\text { documento (E3) }\end{array}$ & $\begin{array}{l}\text { - Apreciação geral } \\
\text { positiva (E1, E2, } \\
\text { E3, E4, E5, E7) } \\
\text {-Incorporação de } \\
\text { inúmeros contribu- } \\
\text { tos e flexibilidade } \\
\text { (E5, E6) } \\
\text { - Inovação e } \\
\text { carácter organiza- } \\
\text { dor da proposta } \\
\text { (E3, E4) }\end{array}$ & - \\
\hline $\begin{array}{l}\text { A. Fenómeno e } \\
\text { necessidades }\end{array}$ & - & - & - & - & - \\
\hline $\begin{array}{l}\text { B. Quadro con- } \\
\text { ceptual/ } \\
\text { objectivos defi- } \\
\text { nidos }\end{array}$ & $\begin{array}{l}\text { - Clareza dos pressupos- } \\
\text { tos orientadores e coe- } \\
\text { rência na integração } \\
\text { teórica (E5) }\end{array}$ & Sim & - & $\begin{array}{l}\text { - Carácter colabo- } \\
\text { rativo do modelo } \\
(\mathrm{E} 5, \mathrm{E} 6, \mathrm{E} 7)\end{array}$ & $\begin{array}{l}\text { - Explicitação dos princí- } \\
\text { pios organizadores do } \\
\text { modelo }(E 5, E 2)\end{array}$ \\
\hline $\begin{array}{l}\text { C. População- } \\
\text {-alvo }\end{array}$ & - & & $\begin{array}{l}\text { - Constituição da } \\
\text { equipa de dois } \\
\text { elementos gesto- } \\
\text { res de caso (E2, } \\
\text { E3) }\end{array}$ & $\begin{array}{l}\text { - Focalização na } \\
\text { família (E4) }\end{array}$ & - \\
\hline
\end{tabular}




\begin{tabular}{|c|c|c|c|c|c|}
\hline & & & & $\begin{array}{l}\text { - Constituição de } \\
\text { equipa de dois } \\
\text { elementos gestores } \\
\text { de caso (E2) }\end{array}$ & \\
\hline $\begin{array}{l}\text { D. Adequação } \\
\text { dos métodos }\end{array}$ & $\begin{array}{l}\text { - Clarificação da especi- } \\
\text { ficidade do modelo em } \\
\text { relação com a articula- } \\
\text { ça com outros serviços } \\
\text { (E1, E2, E3, E4) } \\
\text {-Especificidade do } \\
\text { modelo em casos de } \\
\text { maus tratos (E1, E6) }\end{array}$ & Sim & $\begin{array}{l}\text { - Constituição de } \\
\text { equipa por dois } \\
\text { elementos (E2, } \\
\text { E3) }\end{array}$ & $\begin{array}{l}\text {-Benefício da } \\
\text { intervenção face a } \\
\text { custos da retirada } \\
\text { da criança da famí- } \\
\text { lia }\end{array}$ & $\begin{array}{l}\text { - Explicitação da articula- } \\
\text { ção entre profissio- } \\
\text { nais/serviços e especifici- } \\
\text { dade da proposta }(E 1, E 3)\end{array}$ \\
\hline $\begin{array}{l}\text { E. Adequação } \\
\text { dos recursos }\end{array}$ & $\begin{array}{l}\text { - Constituição da equipa } \\
\text { por dois elementos (E3) } \\
\text { - Número de elementos } \\
\text { na população-alvo (E7) } \\
\text { - Custos específicos do } \\
\text { modelo e relação custo- } \\
\text {-benefício (E4, E7) } \\
\text { - Financiamento (E4, } \\
\text { E6, E7) }\end{array}$ & $\begin{array}{l}\text { Sim } \\
\text { Sim } \\
\text { Não }\end{array}$ & $\begin{array}{l}\text { - Custos financei- } \\
\text { ros (E4) }\end{array}$ & - & - \\
\hline $\begin{array}{l}\text { F. Adequação } \\
\text { dos procedimen- } \\
\text { tos avaliativos }\end{array}$ & $\begin{array}{l}\text { - Informatização dos } \\
\text { procedimentos e docu- } \\
\text { mentos (E3) }\end{array}$ & Sim & - & - & $\begin{array}{l}\text { - Inclusão de indicadores } \\
\text { de progressão sócio- } \\
\text {-económica e outros indi- } \\
\text { cadores atípicos de formas } \\
\text { tradicionais de terapia (E6) } \\
\text { - Inclusão de avaliações } \\
\text { pessoais positivas dos } \\
\text { profissionais em grelhas de } \\
\text { avaliação (E2) }\end{array}$ \\
\hline
\end{tabular}


As dúvidas colocadas pelo painel incidiram, fundamentalmente, sobre a especificidade da proposta do MAIFI e sobre a respectiva articulação com serviços e apoios já existentes (e.g., "como é que este serviço evitará a fragmentação da intervenção?"; "não está muito claro no documento como se faz a intervenção, que não é mais uma intervenção, mas algo que vai ser integrado no resto"; ou "acabam por oferecer um serviço de mediação?"). Na sequência, uma das recomendações inclui a proposta de ser desenhado um mapa esclarecedor da articulação e diferenciação do MAIFI relativamente a outros serviços (e.g., "delinear um bocadinho esse mapa [da articulação entre serviços], acho que seria útil"). No sentido de deixar bem claro o quadro conceptual subjacente ao modelo, o painel sugeriu que se destaquem, na proposta do MAIFI, os seus princípios orientadores ("acho que, se puder, fundá-lo num conjunto de princípios articulados, sabe" "aqui estão os compromissos que organizam o nosso trabalho" (...) "trazê-los mais para o início"; "acho que ganhava se ficasse escrito nos objectivos que se pretende que as famílias ganhem autonomia"). Esta sugestão decorre, não tanto da falta de clareza ou consistência dos mesmos, mas da necessidade de, num documento tão longo e complexo como o que apresenta o MAIFI, tais princípios serem facilmente identificados.

O facto de a equipa destacada para cada caso ser constituída por dois elementos suscitou dúvidas, essencialmente aos académicos nacionais, e foi avaliado, quer como um aspecto positivo (e.g., "eu penso que é enriquecedor serem dois desde que haja articulação entre eles, até porque se calhar um pode ter mais feeling para aquele caso do que o outro"), quer como um aspecto negativo (e.g., "eu acho que a família tem falta de dizer «o meu profissional é este»."), tendo sido referida a importância de haver um profissional de referência.

No que diz respeito à proposta do perfil de competências e do plano de formação dos profissionais do MAIFI, as avaliações foram, na generalidade, positivas, obtendo-se avaliações médias superiores a 4 , conforme pode verificar-se no quadro 4.

O quadro 5 apresenta os resultados da avaliação qualitativa. Menos avaliações são explicitamente apoiadas por mais do que um especialista. A previsão de custos elevados é apontada, mais uma vez, como um aspecto negativo pelos especialistas que representam o ponto de vista das instituições e dos financiadores (e.g., "do ponto de vista dos recursos financeiros. Não obstante a resposta que foi dada tenho muitas dúvidas, porque efectivamente não me parece que seja viável num país como o nosso."; "será dispendioso". $\mathrm{O}$ especialista E1 considerou que a proposta era exequível, embora também tenha referido poderem existir constrangimentos práticos à sua implementação (e.g., "A minha dúvida é mesmo a questão prática, do número de horas de execução do terreno. É mesmo as questões logísticas, as questões práti- 
cas. Podemos ter os profissionais durante este tempo, desde que haja dinheiro (...) [mas] sabemos que a formação implica um esforço pessoal"). Algumas avaliações repetem temas que emergiram a propósito do MAIFI, nomeadamente a necessidade de salientar, durante a formação, os pressupostos organizadores do modelo, como forma de orientar os profissionais e garantir a coerência do seu trabalho ("garanta que, durante a formação, as pessoas têm uma posição a partir da qual operar, uma metáfora organizadora, por oposição a basearem-se em técnicas saídas de diferentes bases epistemológicas").

Quadro 4. Questões para avaliação do perfil de competências, plano de formação e acompanhamento dos profissionais, pontuações médias, mínimas e máximas

\begin{tabular}{|c|c|c|c|}
\hline Questões formuladas & $\begin{array}{l}\text { Pontuação } \\
\text { média }\end{array}$ & $\begin{array}{l}\text { Pontuação } \\
\text { mínima }\end{array}$ & $\begin{array}{l}\text { Pontuação } \\
\text { máxima }\end{array}$ \\
\hline Total das questões & 4.39 & - & - \\
\hline $\begin{array}{l}\text { 1. A proposta é relevante considerando } \\
\text { as necessidades dos profissionais e das } \\
\text { instituições para implementação do } \\
\text { MAIFI? }\end{array}$ & 4.43 & 4 & 5 \\
\hline $\begin{array}{l}\text { 2. A proposta é pertinente tendo em } \\
\text { consideração a formação de base dos } \\
\text { profissionais? }\end{array}$ & 4.14 & 3 & 4 \\
\hline $\begin{array}{l}\text { 3. A proposta é adequada relativamen- } \\
\text { te aos pressupostos teóricos e concep- } \\
\text { tuais que a enquadram e ao MAIFI? }\end{array}$ & 4.57 & 4 & 5 \\
\hline $\begin{array}{l}\text { 4. Os objectivos definidos são perti- } \\
\text { nentes e congruentes com o enqua- } \\
\text { dramento conceptual/teórico do MAI- } \\
\text { FI? }\end{array}$ & 4.57 & 4 & 5 \\
\hline $\begin{array}{l}\text { 5. As estratégias e componentes apre- } \\
\text { sentadas na proposta são adequadas? }\end{array}$ & 4.43 & 4 & 5 \\
\hline $\begin{array}{l}\text { 6. A proposta é exequível do ponto de } \\
\text { vista da implementação no terreno? }\end{array}$ & 4.21 & 3 & 5 \\
\hline
\end{tabular}


Quadro 5. Resultados da avaliação qualitativa da proposta relativa ao perfil de competências do profissional e plano de formação do MAIFI

\begin{tabular}{|c|c|c|c|c|}
\hline $\begin{array}{l}\text { Conteúdos das } \\
\text { dúvidas e } \\
\text { comentários }\end{array}$ & $\begin{array}{c}\text { Resposta às } \\
\text { dúvidas } \\
\text { Sim/Não }\end{array}$ & $\begin{array}{c}\text { Conteúdos } \\
\text { de avalia- } \\
\text { ções negati- } \\
\text { vas }^{1}\end{array}$ & $\begin{array}{c}\text { Conteúdos de } \\
\text { avaliações } \\
\text { positivas }^{1}\end{array}$ & $\begin{array}{l}\text { Conteúdos das } \\
\text { sugestões de } \\
\text { melhoramento }\end{array}$ \\
\hline
\end{tabular}

\begin{tabular}{|c|c|c|c|c|}
\hline $\begin{array}{l}\text { - Abordagem do } \\
\text { carácter integra- } \\
\text { dor do modelo } \\
\text { na formação }\end{array}$ & Sim & $\begin{array}{l}\text { - Previsão de } \\
\text { custos eleva- } \\
\text { dos (E4; E7) }\end{array}$ & $\begin{array}{l}\text { - Exequibilida- } \\
\text { de da proposta } \\
\text { (E1) }\end{array}$ & $\begin{array}{lr}\text { - Salientar } & \text { pres- } \\
\text { supostos } & \text { organi- } \\
\text { zadores } & \text { do } \\
\text { modelo } & \text { que }\end{array}$ \\
\hline (E5) & & $\begin{array}{l}\text { - Necessida- } \\
\text { de de mais }\end{array}$ & & $\begin{array}{l}\text { garantem } \\
\text { coerência }\end{array}$ \\
\hline $\begin{array}{l}\text { - Tempo de } \\
\text { formação vs. } \\
\text { supervisão (E3) }\end{array}$ & Sim & $\begin{array}{l}\text { espaço de } \\
\text { supervisão } \\
\text { (E3) }\end{array}$ & & $\begin{array}{l}\text { formação (E5) } \\
\text {-Melhorar e } \\
\text { aumentar a com- } \\
\text { ponente }\end{array}$ \\
\hline $\begin{array}{l}\text { - Inclusão de } \\
\text { conteúdos cen- } \\
\text { trados no fun- } \\
\text { cionamento das } \\
\text { equipas (E2) }\end{array}$ & & $\begin{array}{l}\text { - Dificulda- } \\
\text { des associa- } \\
\text { das a ques- } \\
\text { tões práticas } \\
\text { logísticas e } \\
\text { exigência } \\
\text { para os pro- } \\
\text { fissionais } \\
\text { (E1) }\end{array}$ & & $\begin{array}{l}\text { supervisão } \\
\text { diversificar for- } \\
\text { mas de supervi- } \\
\text { são (e.g., entre } \\
\text { equipas) (E3) }\end{array}$ \\
\hline $\begin{array}{l}\text { - Custos espe- } \\
\text { cíficos da for- } \\
\text { mação/supervisã } \\
\text { o (E7) }\end{array}$ & Não & $\begin{array}{l}\text { - Necessi- } \\
\text { dade de for- } \\
\text { mação sobre } \\
\text { temas da } \\
\text { protecção } \\
\text { (aspectos } \\
\text { jurídicos) } \\
\text { (E4) }\end{array}$ & & $\begin{array}{l}\text { - Elaborar orça- } \\
\text { mento para a } \\
\text { formação }(\mathrm{E} 7)\end{array}$ \\
\hline
\end{tabular}

Nota: ${ }^{1}$ Entre parêntesis são indicados, de E1 a E7, os especialistas que propuseram ou apoiaram os conteúdos apresentados por outros. 


\section{Conclusão}

O presente estudo visou avaliar, recorrendo a um painel de especialistas, a qualidade do planeamento do Modelo de Avaliação e Intervenção Familiar Integrada (MAIFI), da definição do perfil de competências dos profissionais e do plano de formação e acompanhamento dos mesmos.

A avaliação foi, na generalidade, bastante satisfatória, representando diferentes pontos de vista, desde o profissional do terreno às organizações e aos financiadores, incluindo também a perspectiva de académicos e investigadores. Foram sublinhados, como pontos fortes: 1) a organização de um modelo de avaliação e intervenção familiar que respeite o bem-estar e as opções da família, garantindo, contudo, a segurança e crescimento saudável da criança; 2) o carácter colaborativo do mesmo; 3) o carácter inovador da proposta; 4) a sua dimensão integradora, de diferentes contributos teóricos e estratégias de acção; 5) a apresentação de linhas de orientação claras para os profissionais que estão no terreno; 6) a existência de uma proposta de formação e acompanhamento dos mesmos.

Pela extensão do documento, pela complexidade do trabalho solicitado aos profissionais e pela diversidade e amplitude dos conhecimentos exigidos (no que diz respeito aos modelos teóricos envolvidos e às estratégias de avaliação e intervenção solicitadas), foi recomendado que estivessem sempre muito claros: 1) os pressupostos orientadores do trabalho dos profissionais; 2) as modalidades e objectivos da articulação entre profissionais. Foi também sublinhada a importância do processo de formação e, particularmente, da supervisão dos profissionais que venham a aplicar o MAIFI.

Os custos financeiros da implementação do MAIFI e da formação dos profissionais foram aspectos particularmente realçados por parte de quem financia e representa as instituições sociais, tendo sido salientada a necessidade de serem elaborados, no futuro, cálculos específicos dos custos da implementação do MAIFI e do plano de formação e acompanhamento das equipas.

Os resultados sugerem que existem condições para que o MAIFI possa continuar a ser desenvolvido, implementado e avaliado. O modelo foi avaliado como inovador, podendo ser particularmente organizador da actividade dos CAFAP, no apoio a famílias multi-desafiadas e com crianças e jovens em situação de risco e de perigo. A avaliação conduzida, embora sujeita a limitações, poderá legitimar a adopção do MAIFI por parte dos CAFAP, mas também oferecer uma base para que esta adopção não seja feita de forma acrítica. As sugestões de melhoramento foram tidas em consideração, nomeadamente nas experiências de implementação e divulgação do MAIFI que a primeira autora já realizou. Como resultado da avaliação realizada pelo 
painel, de algumas das suas questões e avaliações mais críticas, foi dada particular atenção à definição clara da especificidade do modelo e à articulação entre diferentes serviços.

Numa altura em que os Centros de Apoio Familiar e Aconselhamento Parental (CAFAP) se encontram ainda sem regulamentação ou legislação de enquadramento, e quando faltam metodologias comuns entre os CAFAP, o MAIFI pode contribuir para colmatar uma lacuna relevante no panorama dos serviços nacionais, bem como para a construção da identidade destes serviços em torno de uma metodologia de avaliação e intervenção especializada, de apoio às Comissões de Protecção de Crianças e Jovens, aos Tribunais e serviços sociais que trabalham com famílias multi-desafiadas em situações de risco ou perigo. Por outro lado, o presente estudo cria condições para que possa ser desenvolvida investigação em torno da sua eficácia e dos processos envolvidos na sua implementação e para que os serviços prestados pelos CAFAP possam afirmar a sua credibilidade e utilidade.

Podem, contudo, ser identificadas várias limitações neste estudo, entre as quais o facto de os avaliadores serem os proponentes do programa. Este facto pode ter dificultado uma condução neutra da reunião de avaliação e uma distribuição equilibrada da atenção dada aos diversos tópicos. Por outro lado, as avaliações reflectem, em última instância, as posições dos especialistas particulares em questão, aspecto que ameaça a validade das mesmas. Estas limitações fazem com que os resultados tenham que ser entendidos, dentro do contexto em que foram obtidos e interpretados, com cautela. Tais limitações reforçam a necessidade de se conduzirem futuras avaliações do programa, com especial enfoque no processo de implementação do MAIFI e resultados ou impacto associados.

\section{Referências}

Andersen, T. (1995). Reflecting processes: Acts of informing and forming. In S. Friedman (Ed.), The reflecting team in action: Collaborative therapy in family therapy (pp. 11-37). New York: The Guilford Press.

Anderson, H., \& Goolishian, H. (1992). The client is the expert: A not-knowing approach to therapy. In S. McNamee \& K. Gergen (Eds.), Social construction and the therapeutic process (pp. 25-39). Newbury Park, CA: Sage.

Armstrong, C., \& Hill, M. (2001). Support services for vulnerable families with young families. Child and Family Social Work, 6, 351-358.

Averch, H. A. (2004). Using expert judgment. In J. S. Wholey, H. P. Hatry \& K. E. Newcomber (Eds.), Handbook of practical program evaluation $\left(2^{\text {nd }}\right.$ ed.) (pp. 292-309). San Franscisco, CA: Jossey-Bass.

Berg, I. K., \& Kelly, S. (2000). Building solutions in child protective services. New York: W. W. Norton \& Company. 
Boyd-Franklin, N., \& Bry, B. H. (2000). Reaching out in family therapy: Home-based, school and community interventions. New York: The Guilford Press.

Carr, A. (2006). Family therapy: Concepts, process and practice $\left(2^{\text {nd }}\right.$ ed.). Chichester: John Wiley and Sons.

Cirillo, S., \& Di Blasio, P. (1997). Niños maltratados: Diagnóstico y terapia familiar ( $2^{\mathrm{a}}$ reimpressión). Barcelona: Paidós.

Coffey, A., \& Atkinson, P. (1996). Making sense of qualitative data: Complementary research strategies. Thousand Oaks, CA: Sage.

Cunningham, P. B., \& Henggeler, S. W. (1999). Engaging multiproblem families in treatment: Lessons learned throughout the development of multisystemic therapy. Family Process, 38, 265-281.

Dallos, R., \& Urry, A. (1999). Abandoning our parents and grandparents: Does social construction mean the end of systemic therapy?. Journal of Family Therapy, 21, 161-186.

De Jong, P., \& Miller, S. D. (1995). How to interview for client strengths. Social Work, 40(6), 729-736.

De Shazer, S. (1991). Putting difference to work. New York: Norton.

Direcção Geral da Segurança Social, da Família e da Criança [DGSSFC/MTSS] (2006). Respostas Sociais-Nomenclaturas/Conceitos. [Versão electrónica] Lisboa: Direcção Geral da Segurança Social, da Família e da Criança. Disponível em http://195.245.197.196/do_publicacoes.asp?tit=Nomenclaturas\& Action=Ver, retirado em Julho 2007.

Frankel, H., \& Frankel, S. (2006). Family therapy, family practice, and child and family poverty: Historical perspectives and recent developments. Journal of Family Social Work, 10(4), 43-80.

Friedman, S. (Ed.) (1993). The new language of change. Constructive collaboration in psychotherapy. New York: The Guilford Press.

Herman, J. L., Morris, L. L., \& Fitz-Gibbon, C. T. (1987). Evaluator's handbook. Newbury Park, CA: Sage Publications.

Hoagwood, K. E. (2005). Family-based services in children's mental health: A research review and synthesis. Journal of Child Psychology and Psychiatry, 46(7), 690-713.

Hoffman, L. (1998). Setting aside the model in family therapy. In M. F. Hoyt (Ed.), The handbook of constructive therapies: Innovative approaches from leading practitioners (pp. 100-115). San Francisco: Jossey-Bass.

Hoffman, S. (2005). Ten years on: Lessons learned from the institutional evaluation programme [electronic version]. Brussels: European University Association. Disponível em: http://www.eua.be/, retirado em Julho 2007.

Jones, J. (1995). Qualitative research: Consensus methods for medical and health services research. British Medical Journal, 311, 376-380.

Kröger, C., Winter, H., \& Shaw, R. (1998). Linhas orientadoras para avaliação de acções de prevenção da toxicodependência. Lisboa: Observatório Europeu da Droga e Toxicodependência.

Madsen, W. C. (2007). Collaborative therapy with multi-stressed families ( $2^{\text {nd }}$ ed.). New York: The Guilford Press. 
Manalo, V., \& Meezan, W. (2000). Toward building a typology for the evaluation of services in family support programs. Child Welfare, LXXIX(4), 405-429.

Marsh, J. C., Ryan, J., Choi, S., \& Testa, M. F. (2006). Integrated services for families with multiple problems: Obstacles to family reunification. Children and Youth Services Review, 28, 1074-1087.

McDonald, D., Bammer, G., \& Deane, P. (2009). Research integration using dialogue methods. Canberra: ANU EPress.

Melo, A. T. (2008). Apresentação de uma proposta de um modelo global de organização dos CAFAP, no Modelo de Avaliação e Intervenção Familiar Integrada e respectivo plano perfil de competências e plano de formação dos profissionais. Coimbra: Faculdade de Psicologia e de Ciências da Educação da Universidade de Coimbra. Disponível com os autores.

Melo, A. T., \& Alarcão, M. (2010b). Integrated Family Assessment and Intervention Model: A collaborative approach for supporting multi-challenged families with at-risk or maltreated children. Manuscrito em preparação.

Melo, A. T., \& Alarcão, M. (2009a). Centros de apoio familiar e aconselhamento parental: Proposta de um modelo global de organização. Psicologia \& Sociedade, 21(1), 55-64.

Melo, A. T., \& Alarcão, M. (2009b). Avaliação de uma proposta de modelo global de organização para os centros de apoio familiar e aconselhamento parental. Manuscrito submetido para publicação.

Melo, A. T., \& Alarcão, M. (2010b). From multi-problem to multi-challenged families: A multiple challenges comprehensive model. Manuscrito em preparação.

Minuchin, P., Colapinto, J., \& Minuchin, S. (2007). Working with families of the poor $\left(2^{\text {nd }}\right.$ ed.). New York: The Guilford Press.

Patton, M. Q. (1997). Utilization-focused evaluation: The new century text $\left(3^{\text {rd }}\right.$ ed.). London: Sage Publications.

Patton, M. Q. (2002). Qualitative research and evaluation methods ( $3^{\text {rd }}$ ed.). Thousand Oaks, CA: Sage Publications.

Pecora, P. J., Seelig, W. R., Zirps, F. A., \& Davis, S. M. (Eds.) (1996). Quality improvement and evaluation in child and family services. Managing into the next century. Washington, DC: CWLA Press.

Posavac, E. I., \& Carey, R. G. (2003). Program evaluation: Methods and case studies. $\left(6^{\text {th }}\right.$ ed). New Jersey: Prentice Hall.

Robson, C. (1993). Real world research: A resource for social scientists and practitioner-researchers. Oxford: Blackwell Publishers.

Rojano, R. (2004). The practice of community family therapy. Family Process, 43, 59-77.

Rossi, P. H., Lipsey, M. W., \& Freeman, H. E. (2004). Evaluation: A systematic approach $\left(7^{\text {th }}\right.$ ed.). Thousand Oaks, CA: Sage Publications.

Saleebey, D. (Ed.) (2002). The strengths perspective in social work practice $\left(3^{\mathrm{rd}}\right.$ ed.). Boston: Allyn \& Bacon.

Silverman, D. (2006). Interpreting qualitative data: Methods for analyzing talk, text and interaction $\left(3^{\text {rd }}\right.$ ed.). London: Sage Publications. 
Sluzki, C. E. (1992). Transformations: A blueprint for narrative changes in therapy. Family Process, 31, 217-230.

Sousa, L. (2005). Famílias multiproblemáticas. Coimbra: Quarteto editora.

Turnell, A., \& Edwards, S. (1999). Signs of safety: A solution and safety-oriented approach to child protection casework. New York: W. W. Norton \& Company.

Vetere, A., \& Dallos, R. (2003). Working systemically with families: Formulation, intervention and evaluation. London: Karnac Books.

Walsh, T. (2006). Two sides of the same coin: Ambiguity and complexity in child protection work. Journal of Systemic Therapies, 25(2), 38-49.

White, M. (2007). Maps of narrative practice. New York: W. W. Norton \& Company.

Woodford, M. S., Bordeau, W. C., \& Alderfer, C. C. (2006). Home-based service delivery: Introducing family counsellors in training to the home as a therapeutic milieu. The Family Journal: Counselling and Therapy for Couples and Families, 14 (3), 240-244. 